\title{
Generalized Fractional Order Derivatives for Products and Quotients
}

\author{
Ali Karci \\ Department of Computer Engineering, Faculty of Engineering, İnönü University, Malatya, Turkey
}

Email address:

adresverme@gmail.com, ali.karci@inonu.edu.tr

To cite this article:

Ali Karci. Generalized Fractional Order Derivatives for Products and Quotients. Science Innovation. Vol. 3, No. 5, 2015 , pp. 58-62. doi: 10.11648/j.si.20150305.13

\begin{abstract}
The concept of fractional order derivative (FOD) can be found in extensive range of many different subject areas. For this reason, the concept of FOD should be examined in wide range. There are lots of methods about FOD in the literature; however, none of them are FOD methods. Since all of them are curve fitting or curve approximation methods. In fact, the methods used in the literature are not FOD methods; they are approximation methods. In this paper, we redefined FOD for product and quotient. The obtained definition is same as classical derivative definition in case of fractional order is equal to 1 . FOD of products and quotients were handled in this paper with some applications. The properties of both theorems were analysed in this paper.
\end{abstract}

Keywords: Fractional Order Derivatives, Derivative of Product, Derivative of Quotient

\section{Introduction}

Fractional calculus is an old calculus, but not very popular amongst science and engineering community. The fractional derivative is not a local property in mathematics. The fractional derivatives may translate the reality of nature better as similar to fuzzy logic. Therefore to make this subject available as popular subject to science and engineering community, the fractional derivative must be redefined analytically. The description of nature in this way may make us understand the nature well.

The FODs are different approaches instead of classical derivatives. There are a lot of studies on this subject. The most of these studies have used Euler, Riemann-Liouville and Caputo FODs. Due to this case, this study focused on Euler, Riemann-Liouville and Caputo FODs.

Some of studies on the FODs can be summarized as follows:

A minimization problem with a Lagrangian that depends on the left Riemann-Liouville fractional derivative was considered in [1] such as finite differences, as a subclass of direct methods in the calculus of variations, consist in discretizing the objective functional using appropriate approximations for derivatives that appear in the problem. There is a study on fractional extensions of the classical Jacobi polynomials [2], and fractional order Rodrigues' type representation formula. By means of the Riemann-Liouville operator of fractional calculus, new g-Jacobi functions were defined, some of their properties were given and compared with the corresponding properties of the classical Jacobi polynomials [2]. There is another study on the discussion of theory of fractional powers of operators on an arbitrary Frechet space, and the authors of this study obtained multivariable fractional integrals and derivatives defined on certain space of test functions and generalized functions [3].

Differential equations of fractional order appear in many applications in physics, chemistry and engineering [4]. There is a requirement for an effective and easy-to-use method for solving such equations. Bataineh et al used series solutions of the fractional differential equations using the homotopy analysis method [4]. Many recently developed models in areas like viscoelasticity, electrochemistry, diffusion processes, etc. are formulated in terms of derivatives (and integrals) of fractional (non-integer) order [5]. There is a collection of numerical algorithms for the solution of the various problems arising in derivatives of fractional order [5].

The fractional calculus is used to model various different phenomena in nature but due to the non-local property of the fractional derivatives, it still remains a lot of improvements in the present numerical approaches [6]. There are some 
approaches based on piecewise interpolation for fractional calculus, and some improvement based on the Simpson method for the fractional differential equations [6]. There is a study on fractional order iterative learning control including many theoretical and experimental results, and these results shown the improvement of transient and steady-state performances [7].

Recently, many models are formulated in terms of fractional derivatives, such as in control processing, viscoelasticity, signal processing, and anomalous diffusion, and authors of this paper studied the important properties of the Riemann-Liouville derivative, one of mostly used fractional derivatives [8]. The philosophy of integer order sliding mode control is valid also for the systems represented by fractional order operators [9].

Here is just a small part of the work in this area is given. Except these, there are numerous studies. This area of work can be divided into two groups such as mathematical theory of FODs and the applications of FODs. There are important errors and deficiencies in both groups of studies. Due to this case, the FOD concept was redefined in $[11,12,13,14]$.

In this study, it is focused on the FODs of product and quotient. This paper is organized as follow. Section 2 describes the FODs of any function, product and quotient. Section 3 describes the applications of FODs. Finally, section 4 describes the conclusion of this study.

\section{Fractional Order Derivatives}

\subsection{Analytic Definition for Fractional Order Derivatives}

The fractional order derivative (FOD) concept was redefined in $[11,12,13,14]$ and the reason of definition and definition can be given as follow.

The meaning of derivative is the rate of change in the dependent variable versus the changes in the independent variables. There are many studies on this subject; however, all methods in the literature consist of some deficiencies and misleading information. At this aim, the derivative of $f(x)=c x^{0}$ is that

$$
\lim _{h \rightarrow 0} \frac{f(x+h)-f(x)}{(x+h)-x}=\lim _{h \rightarrow 0} \frac{c-c}{h}=0
$$

In the case of identity function is

$$
\lim _{h \rightarrow 0} \frac{f(x+h)-f(x)}{(x+h)-x}=\lim _{h \rightarrow 0} \frac{(x+h)-x}{(x+h)-x}=1
$$

So, the definition for FOD can be considered as follow.

Definition 1: $[11,12,13,14] f(x): R \rightarrow R$ is a function, $\alpha \in R$ and the FOD can be considered as follows.

$$
f^{(\alpha)}(x)=\lim _{h \rightarrow 0} \frac{f^{\alpha}(x+h)-f^{\alpha}(x)}{(x+h)^{\alpha}-x^{\alpha}}
$$

In the case of very small value of $h$, the limit in the Definition 1 concluded in indefinite limit.

$$
f^{(\alpha)}(x)=\lim _{h \rightarrow 0} \frac{f^{\alpha}(x+h)-f^{\alpha}(x)}{(x+h)^{\alpha}-x^{\alpha}}=\frac{0}{0}
$$

In this case, the method used for indefinite limit (such as L'Hospital method) can be used, and the fractional order derivative can be redefined as follows.

Definition 2: $[11,12,13,14]$ Assume that $f(x): R \rightarrow R$ is a function, $\alpha \in R$ and $L$ (.) be a L'Hospital process. The FOD of $f(x)$ is

$$
\begin{aligned}
& f^{(\alpha)}(x)=\lim _{h \rightarrow 0} L\left(\frac{f^{\alpha}(x+h)-f^{\alpha}(x)}{(x+h)^{\alpha}-x^{\alpha}}\right) \\
& =\lim _{h \rightarrow 0} \frac{d\left(f^{\alpha}(x+h)-f^{\alpha}(x)\right)}{\frac{d h}{d\left((x+h)^{\alpha}-x^{\alpha}\right)}} \frac{d h}{d h}
\end{aligned}
$$

The existence of this definition can be verified by the Theorem 1.

Theorem 1: Assume that $f(x): R \rightarrow R$ is a continue function and $\alpha \in R$. The limit

$$
M=\lim _{h \rightarrow 0} L\left(\frac{f^{\alpha}(x+h)-f^{\alpha}(x)}{(x+h)^{\alpha}-x^{\alpha}}\right)
$$

exists and is finite.

Proof: $\quad \mathrm{f}^{(\alpha)}(\mathrm{x}+\mathrm{h})-\mathrm{f}^{(\alpha)}(\mathrm{x})<\infty \quad$ and $\quad(\mathrm{x}+\mathrm{h})^{(\alpha)}-\mathrm{x}^{(\alpha)}<\infty$. $f^{(\alpha)}(x+h)-f^{(\alpha)}(x) \neq 0$ and $(x+h)^{(\alpha)}-x^{(\alpha)} \neq 0$. Then

$$
\begin{gathered}
\lim _{h \rightarrow 0} L\left(\frac{f^{\alpha}(x+h)-f^{\alpha}(x)}{(x+h)^{\alpha}-x^{\alpha}}\right)<\infty \\
\text { And } \lim _{h \rightarrow 0} L\left(\frac{f^{\alpha}(x+h)-f^{\alpha}(x)}{(x+h)^{\alpha}-x^{\alpha}}\right)=M<\infty .
\end{gathered}
$$

The definition of derivative has been handled for $\alpha=1$ until today. While $\alpha=1$ for definition 2 , the obtained results are same to results of classical derivative. While $\alpha=2$, the FOD is different from $\frac{d}{d x}\left(\frac{d f(x)}{d x}\right)$. Since

$$
\begin{aligned}
\frac{d}{d x}\left(\frac{d f(x)}{d x}\right)= & \lim _{h \rightarrow 0} \frac{f^{1}(x+h)-f^{1}(x)}{h} \\
& =\lim _{h \rightarrow 0} \frac{f(x+2 h)-2 f(x+h)+f(x)}{h^{2}}
\end{aligned}
$$

The fractional order derivative definition can be demonstrated that it obtained same results as classical derivative definition for $\alpha=1$.

\subsection{FODs of Products and Quotients}

The new derivation definition can be applied to products and quotients. In order to give the FOD of real functions, the derivatives of products and quotients must be given for $\alpha=1$. Assume that $h(x)=f(x) g(x)$, and the derivative of $h(x)$ is $h^{(\alpha)}(x)=h^{(1)}(x)=h^{\prime}(x)[10]$. 


$$
\begin{aligned}
& h^{\prime}(x)=\lim _{h \rightarrow 0} \frac{h(x+h)-h(x)}{h} \\
& =\lim _{h \rightarrow 0} \frac{f(x+h) g(x+h)-f(x) g(x)}{h} \\
& =\lim _{h \rightarrow 0}\left(f(x+h) \frac{g(x+h)-g(x)}{h}\right) \\
& =\lim _{h \rightarrow 0} f(x+h) \lim _{h \rightarrow 0} \frac{g(x+h)-g(x)}{h} \\
& +\lim _{h \rightarrow 0} g(x+h) \lim _{h \rightarrow 0} \frac{f(x+h)-f(x)}{h} \\
& =f(x) g^{\prime}(x)+g(x) f^{\prime}(x)
\end{aligned}
$$

The same process can be illustrated for quotient, and assume that $h(x)=\frac{f(x)}{h(x)}$. The derivative of $h(x)[10]$ is

$$
\begin{aligned}
& h^{\prime}(x)=\lim _{h \rightarrow 0} \frac{\frac{f(x+h)}{g(x+h)}-\frac{f(x)}{g(x)}}{h} \\
& =\lim _{h \rightarrow 0} \frac{f(x+h) g(x)-f(x) g(x+h)}{h g(x+h) g(x)} \\
& =\lim _{h \rightarrow 0} \frac{g(x) \frac{f(x+h)-f(x)}{h}-f(x) \frac{g(x+h)-g(x)}{h}}{g(x+h) g(x)} \\
& =\frac{f^{\prime}(x) g(x)-f(x) g^{\prime}(x)}{g^{2}(x)}
\end{aligned}
$$

By using this knowledge from literature, the FOD of product and quotient can be rephrased in Theorems 2 and 3 .

Theorem 2: Assume that $f(x), g(x): R \rightarrow R$ are continuous functions, $\alpha \in R$ and $h(x)=f(x) g(x)$. The FOD of $h(x)$ is as follows.

$$
h^{(\alpha)}(x)=\left(\frac{f(x) g(x)}{x}\right)^{\alpha-1}\left(f^{\prime}(x) g(x)+f(x) g^{\prime}(x)\right)
$$

Proof: $\mathrm{f}(\mathrm{x})$ and $\mathrm{g}(\mathrm{x})$ are real functions, $\mathrm{so}, \mathrm{h}(\mathrm{x})$ is also a real function. FOD of $\mathrm{h}(\mathrm{x})$

$$
\begin{aligned}
h^{(\alpha)} & =\lim _{h \rightarrow 0} L\left(\frac{(f(x+h) g(x+h))^{\alpha}-f^{\alpha}(x) g^{\alpha}(x)}{(x+h)^{\alpha}-x^{\alpha}}\right) \\
& =\lim _{h \rightarrow 0}\left(\frac{\alpha f^{\prime}(x+h) f(x+h)^{\alpha-1} g^{\alpha}(x+h)}{\alpha(x+h)^{\alpha-1}}+\right) \\
& \left.=\frac{\alpha f^{\alpha}(x+h) g^{\prime}(x+h) g^{\alpha-1}(x+h)}{\alpha(x+h)^{\alpha-1}}\right)
\end{aligned}
$$

$$
=\left(\frac{f(x) g(x)}{x}\right)^{\alpha-1}\left(f^{\prime}(x) g(x)+f(x) g^{\prime}(x)\right)
$$

Theorem 3: Assume that $f(x), g(x): R \rightarrow R$ is a continue functions, $\alpha \in R$ and $\mathrm{h}(\mathrm{x})=\frac{\mathrm{f}(\mathrm{x})}{\mathrm{g}(\mathrm{x})}$. The FOD of $h(x)$ is as follows.

$$
h^{(\alpha)}(x)=\left(\frac{f(x)}{x g(x)}\right)^{\alpha-1} \frac{f^{\prime}(x) g(x)-f(x) g^{\prime}(x)}{g^{2}(x)} .
$$

Proof: $\mathrm{f}(\mathrm{x})$ and $\mathrm{g}(\mathrm{x})$ are real functions, $\mathrm{so}, \mathrm{h}(\mathrm{x})$ is also a real function. FOD of $\mathrm{h}(\mathrm{x})$

$$
\begin{aligned}
h^{(\alpha)}(x) & =\lim _{h \rightarrow 0} L\left(\frac{\left.\left(\frac{f(x+h)}{g(x+h)}\right)^{\alpha}-\left(\frac{f(x)}{g(x)}\right)^{\alpha}\right)}{(x+h)^{\alpha}-x^{\alpha}}\right) \\
& =\lim _{h \rightarrow 0} L\left(\frac{f^{\alpha}(x+h) g^{\alpha}(x)-f^{\alpha}(x) g^{\alpha}(x+h)}{\left((x+h)^{\alpha}-x^{\alpha}\right) g^{\alpha}(x+h) g^{\alpha}(x)}\right) \\
& =\lim _{h \rightarrow 0}\left(\frac{\alpha f^{\prime}(x+h) f^{\alpha-1}(x+h) g^{\alpha}(x)}{\Psi}\right) \\
+ & \lim _{h \rightarrow 0}\left(\frac{-f^{\alpha}(x) \alpha g^{\prime}(x+h) g^{\alpha-1}(x+h)}{\Psi}\right) \\
= & \frac{g^{\alpha-1}\left(f^{\prime}(x) f^{\alpha-1}(x) g(x)-f^{\alpha}(x) g^{\prime}(x)\right)}{x^{\alpha-1} g^{\alpha-1}(x) g^{\alpha+1}(x)} \\
= & \frac{f^{\prime}(x) f^{\alpha-1}(x) g(x)-f^{\alpha}(x) g^{\prime}(x)}{x^{\alpha-1} g^{\alpha+1}(x)} \\
+ & \left((x+h)^{\alpha}-x^{\alpha}\right) g^{\alpha}(x) \alpha g^{\prime}(x+h) g^{\alpha-1}(x+h) \\
= & \left(\frac{f(x)}{x g(x)}\right)^{\alpha-1} \frac{f^{\prime}(x) g(x)-f(x) g^{\prime}(x)}{g^{2}(x)} \\
& \left(x g^{\alpha}\right)
\end{aligned}
$$

The results of theorem 2 and theorem 3 can be used for a closed loop form system. In order to apply FOD to closed loop system, the integration must be redefined in case of FOD.

Theorem 4: Assume that $f(x)$ is a continuous function, $\alpha \in R$ and FOD of $f(x)$ is $f^{(\alpha)}(x)$. The fractional order integral (FOI) of $f^{(\alpha)}(x)$ is

$$
f(x)=\int f^{(\alpha)}(x) d x^{\alpha}=\sqrt[\alpha-1]{\frac{f^{(\alpha)}(x) x^{\alpha-1}}{f^{\prime}(x)}}
$$

Proof: The FOD of $\mathrm{f}(\mathrm{x})$ is $\mathrm{f}^{(\alpha)}(\mathrm{x})$, and $\int f^{(\alpha)}(x) d x^{\alpha}$ represents the FOI of any function and $f^{(\alpha)}(x)=\frac{f^{\prime}(x) f^{\alpha-1}(x)}{x^{\alpha-1}}$

The FOI of $f^{(\alpha)}(x)$ is 


$$
f(x)=\sqrt[\alpha-1]{\frac{\frac{f^{\prime}(x) f^{\alpha-1}(x)}{x^{\alpha-1}} x^{\alpha-1}}{f^{\prime}(x)}}=\sqrt[\alpha-1]{f^{\alpha-1}(x)} .
$$

FOI of any function is different from classical integration, and the mathematical term $1 / \mathrm{x}$ means integration in the closed loop system modeling, however, the mathematical term $1 / x$ is just a mathematical term in FOI, not integration.

Theorem 5: Assume that $\mathrm{f}(\mathrm{x})$ and $\mathrm{g}(\mathrm{x})$ are monotonic increasing functions and $\alpha_{1}, \alpha_{2}, \ldots, \alpha_{n}$ and $\alpha_{1} \leq \alpha_{2} \leq \ldots \leq \alpha_{n}$. Then $(f(x) g(x))^{\left(\alpha_{1}\right)} \leq(f(x) g(x))^{\left(\alpha_{2}\right)} \leq \cdots \leq(f(x) g(x))^{\left(\alpha_{n}\right)}$.

Proof: Assume that $\mathrm{f}(\mathrm{x})$ and $\mathrm{g}(\mathrm{x})$ are monotonic increasing functions, so, $\mathrm{f}(\mathrm{x}) \mathrm{g}(\mathrm{x})$ is also a monotonic increasing function. It was shown that in theorem 2, the fractional order derivative of product is

$$
h^{(\alpha)}(x)=\left(\frac{f(x) g(x)}{x}\right)^{\alpha-1}\left(f^{\prime}(x) g(x)+f(x) g^{\prime}(x)\right)
$$

The term $f^{\prime}(x) g(x)+f(x) g^{\prime}(x)$ is same in all orders of fractional order derivatives. So, the term $\left(\frac{f(x) g(x)}{x}\right)^{\alpha-1}$ determines the relation $\leq$ between two or more different orders fractional order derivatives. If $\alpha_{i} \leq \alpha_{j}$ for any $1 \leq i, j \leq n$, and $i \neq j$, then $\left(\frac{f(x) g(x)}{x}\right)^{\alpha_{i}-1} \leq\left(\frac{f(x) g(x)}{x}\right)^{\alpha_{j}-1}$.

The similar comments can be drafted for monotonic decreasing functions.

Theorem 6: Assume that $\mathrm{f}(\mathrm{x})$ and $\mathrm{g}(\mathrm{x})$ are monotonic decreasing functions and $\alpha_{1}, \alpha_{2}, \ldots, \alpha_{n}$ and $\alpha_{1} \geq \alpha_{2} \geq \ldots \geq \alpha_{n}$. Then $(f(x) g(x))^{\left(\alpha_{1}\right)} \geq(f(x) g(x))^{\left(\alpha_{2}\right)} \geq \cdots \geq(f(x) g(x))^{\left(\alpha_{n}\right)}$.

Proof: Assume that $\mathrm{f}(\mathrm{x})$ and $\mathrm{g}(\mathrm{x})$ are monotonic decreasing functions, so, $f(x) g(x)$ is also a monotonic decreasing function. It was shown that in theorem 2 , the fractional order derivative of product is

$$
h^{(\alpha)}(x)=\left(\frac{f(x) g(x)}{x}\right)^{\alpha-1}\left(f^{\prime}(x) g(x)+f(x) g^{\prime}(x)\right)
$$

The term $f^{\prime}(x) g(x)+f(x) g^{\prime}(x)$ is same in all orders of fractional order derivatives. So, the term $\left(\frac{f(x) g(x)}{x}\right)^{\alpha-1}$ determines the relation $\geq$ between two or more different orders fractional order derivatives. If $\alpha_{i} \leq \alpha_{j}$ for any $1 \leq i, j \leq n$, and $i \neq j$, then $\left(\frac{f(x) g(x)}{x}\right)^{\alpha_{i}-1} \geq\left(\frac{f(x) g(x)}{x}\right)^{\alpha_{j}-1}$.

If any function is a monotonic increasing and the other is a monotonic decreasing, how we can determine the less than or equal relationships between different orders fractional order derivatives. The theorem 7 determines this case.

Theorem 7: Assume that $\mathrm{f}(\mathrm{x})$ is a monotonic increasing function, and $\mathrm{g}(\mathrm{x})$ is a monotonic decreasing function. Let $\mathrm{h}(\mathrm{x})=\mathrm{f}(\mathrm{x}) \mathrm{g}(\mathrm{x})$ and $\alpha_{\mathrm{i}} \leq \alpha_{\mathrm{j}}$, and there are two cases.

a) If $\mathrm{h}(\mathrm{x})$ is a monotonic increasing function, then $h^{\left(\alpha_{i}\right)}(x) \leq h^{\left(\alpha_{j}\right)}(x)$.

b) If $\mathrm{h}(\mathrm{x})$ is a monotonic decreasing function, then $h^{\left(\alpha_{i}\right)}(x) \geq h^{\left(\alpha_{j}\right)}(x)$.

c) Proof: The proofs of Theorem 5 and Theorem 6 can be used for proving this theorem.

d) The fractional order derivative depends on $\mathrm{h}(\mathrm{x})$ where $h(x)$ is the quotient of $f(x)$ and $g(x)$. If $h(x)$ is a monotonic increasing function and $\alpha \mathrm{i} \leq \alpha \mathrm{j}$, then $h^{\left(\alpha_{i}\right)}(x) \leq h^{\left(\alpha_{j}\right)}(x)$. If $\mathrm{h}(\mathrm{x})$ is a monotonic decreasing function and $\alpha \mathrm{i} \leq \alpha \mathrm{j}$, then $h^{\left(\alpha_{i}\right)}(x) \geq h^{\left(\alpha_{j}\right)}(x)$.

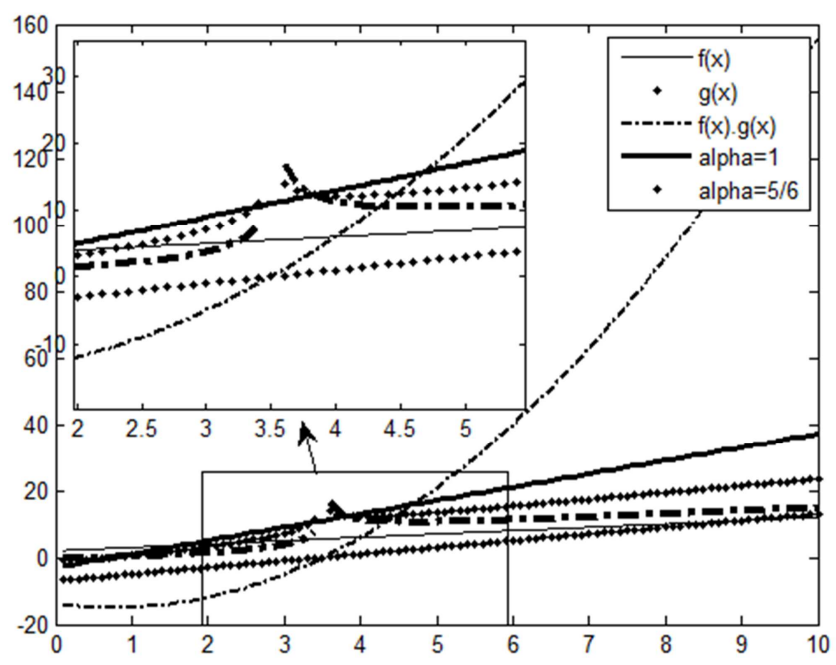

Figure 1. Application of FOD to product for different $\alpha$ values.

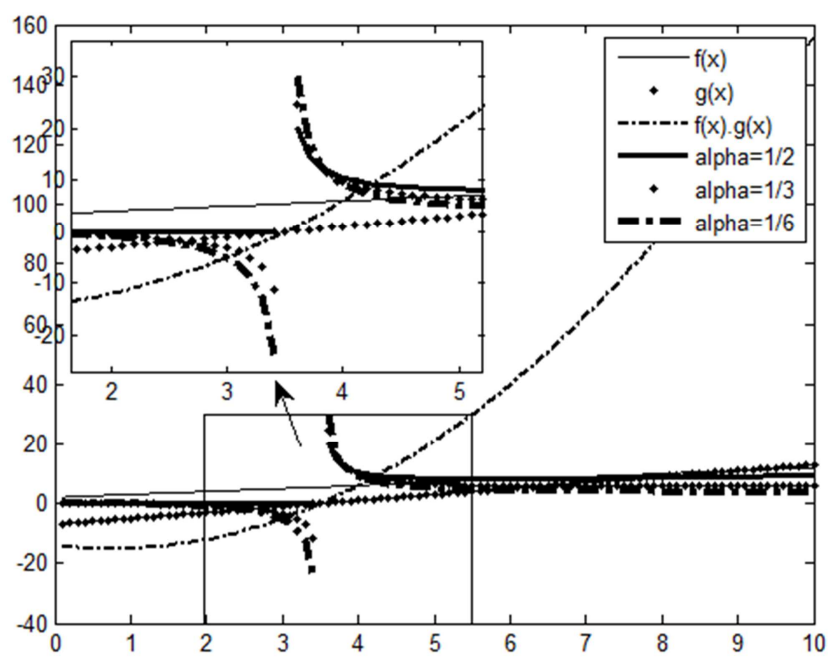

Figure 2. Application of FOD to product for different $\alpha$ values.

\section{Applications of FODs}

In this section, the applications of FOD of product and quotient are handled. Assume that $f(x)=x+2$ is a monotonic increasing function and $\mathrm{g}(\mathrm{x})=2 \mathrm{x}-7$ is also a monotonic increasing function, so $\mathrm{f}(\mathrm{x}) \mathrm{g}(\mathrm{x})$ is also monotonic increasing 
function. Figure 1 and Figure 2 depict the application of FOD to product. Figure 1 and Figure 2 depict the cases in Theorem 5 and Theorem 6.

\section{Conclusions}

The FOD concept is an important concept to make development in mathematics. Due to this reason, the FODs of product and quotient were verified and their applications to some functions were implemented in this study. It can be seen from the proves of Theorem 2 and Theorem 3 that when the order of derivation is 1 , the result of derivation is same as classical derivation; however when order is different from1, the result contains a coefficient for each case. The properties of FOD for product and quotient were handled in this paper.

\section{References}

[1] S. Pooseh, R. Almeida, D. F. M. Torres, Discrete direct methods in the fractional calculus of variations, Computers and Mathematics with Applications, doi:10.1016/j.camwa.2013.01.045.

[2] S. P. Mirevski, L. Boyadjiev, R. Scherer, On the Riemann-Liouville Fractional Calculus, g-Jacobi Functions and F. Gauss Functions, Applied Mathematics and Computation, 187; 315-325 (2007).

[3] S. E. Schiavone, W. Lamb, A Fractional Power Approach to Fractional Calculus, Journal of Mathematical Analysis and Applications, 149; 377-401 (1990).

[4] A. S. Bataineh A. K. Alomari M. S. M. Noorani I. Hashim R. Nazar, Series Solutions of Systems of Nonlinear Fractional Differential Equations, Acta Applied Mathematics, 105; 189-198 (2009).
[5] K. Diethelm, N. J. Ford, A. D. Freed, Yu Luchko, Algorithms for the Fractional Calculus: A Selection of Numerical Methdos, Computer Methods in Applied Mechanics and Engineering, 194; 743-773 (2005).

[6] C. Li, A. Chen, J. Ye, Numerical Approaches to Fractional Calculus and Fractional Ordinary Differential Equation, Journal of Computational Physics, 230; 3352-3368 (2011).

[7] Y. Li, Y. Q. Chen, H.-S. Ahn, G. Tian, A Survey on Fractional-Order Iterative Learning Control, Journal of Optimal Theory and Applications, 156; 127-140 (2013).

[8] C. Li, D. Qian, Y.-Q. Chen, On Riemann-Liouville and Caputo Derivatives, Discrete Dynamics in Nature and Society, 2011, DOI: $10.1155 / 2011 / 562494$.

[9] M. Ö. Efe, Fractional Order Sliding Mode Control with Reaching Law Approach, Turkish Journal of Electrical Engineering \& Computer Science, 18; $731-747$ (2010).

[10] E. A. Swokowski, Calculus with Analytic Geometry, Prindle-Weber \& Schmidt, p: 87-110, (1983).

[11] A. Karc1, Kesirli Türev için Yapılan Tanımlamaların Eksiklikleri ve Yeni Yaklaşım, TOK-2013 Turkish Automatic Control National Meeting and Exhibition.

[12] A. Karc1, Generalized Fractional Order Derivatives, Its Properties and Applications, ArXiv: 1306. 5672 General Mathematics.

[13] A. Karc1, A.Karadoğan, "Fractional Order Derivative and Relationship between Derivative and Complex Functions", IECMSA-2013:2 $2^{\text {nd }}$ International Eurasian Conference on Mathematical Sciences and Applications, Sarajevo, Bosnia and Herzogovina, p: 55-56, Aug. 26-29, 2013.

[14] A. Karc1, "A New Approach for Fractional Order Derivative and Its Applications", Universal Journal of Engineering Sciences, 1(3): 110-117, 2013. 